\title{
Energy Stable High Order Finite Difference Methods for Hyperbolic Equations in Moving Coordinate Systems
}

\author{
Samira Nikkar and Jan Nordström
}

\section{Linköping University Post Print}

\section{Tweet}

N.B.: When citing this work, cite the original article.

Original Publication:

Samira Nikkar and Jan Nordström, Energy Stable High Order Finite Difference Methods for Hyperbolic Equations in Moving Coordinate Systems, 2013, AIAA Aerospace Sciences Fluid Sciences Event, 1-14.

http://dx.doi.org/10.2514/6.2013-2579

From the 21st AIAA Computational Fluid Dynamics Conference 24 - 27 June 2013 San Diego, California

Postprint available at: Linköping University Electronic Press

http://urn.kb.se/resolve?urn=urn:nbn:se:liu:diva-96879 


\title{
Energy Stable High Order Finite Difference Methods for Hyperbolic Equations in Moving Coordinate Systems
}

\author{
Samira Nikkar* \\ Linköping University, Linköping, Sweden
}

\author{
Jan Nordström ${ }^{\dagger}$ \\ Linköping University, Linköping, Sweden
}

\begin{abstract}
A time-dependent coordinate transformation of a constant coefficient hyperbolic equation which results in a variable coefficient problem is considered. By using the energy method, we derive well-posed boundary conditions for the continuous problem. It is shown that the number of boundary conditions depend on the coordinate transformation. By using Summation-by-Parts (SBP) operators for the space discretization and weak boundary conditions, an energy stable finite difference scheme is obtained. We also show how to construct a time-dependent penalty formulation that automatically imposes the right number of boundary conditions. Numerical calculations corroborate the stability and accuracy of the approximations.
\end{abstract}

\section{Nomenclature}

$u \quad$ Dependent variable

$t \quad$ Time

$x, y$ Space coordinate

a Advection speed

$\xi, \eta \quad$ Transformed space coordinate

$a^{(\xi)} \quad$ Advection speed due to moving coordinate system

$v \quad$ Numerical solution

$N$, Number of grid points

$i, j \quad$ Variable number

$\sigma \quad$ Penalty coefficient

$p \quad$ Convergence rate

\section{Introduction}

High order finite difference methods together with summation-by-parts operators and weak implementation of boundary conditions can efficiently and reliably handle large problems on structured grids for reasonably smooth geometries. ${ }^{5,6,9,10,12-15}$ The main reason to use weak boundary procedures (the term boundary condition refers to the continuous problem and boundary procedure is assigned to the numerical implementation of the boundary condition) together with summation-by-parts operators and the energy method is the fact that with this combination, provable stable schemes can be constructed.

The developments described above have so far dealt mostly with steady meshes while computing flowfields around moving objects involves time-dependent meshes. ${ }^{1,2,6}$ We have previously treated the problems with steady curvilinear coordinate transformations. ${ }^{6,14,15}$ In this paper we take the next step, which is the treatment of moving meshes in curvilinear coordinate systems in combination with summation-by-parts operators and weak boundary procedures.

${ }^{*}$ PhD student, Department of Mathematics, Linköping University of Sweden, samira.nikkar@liu.se

${ }^{\dagger}$ Professor, Department of Mathematics, Linköping University, jan.nordstrom@liu.se 


\section{The continuous problem}

\section{A. The one dimensional problem}

Consider the following one dimensional advection equation in a moving domain,

$$
u_{t}+a u_{x}=0, \quad x_{0}(t) \leq x \leq x_{1}(t), \quad t \geq 0,
$$

where $\mathrm{u}$ is the dependent variable and $a$ is the constant wave speed. We transform the moving domain into a fixed domian such that $0 \leq \xi \leq 1$ and $\tau=t$ by

$$
x=x(\xi, \tau), \quad \xi=\xi(x, t) .
$$

The chain-rule is employed to interpret equation (1) in the fixed coordinate frame as

$$
u_{\tau}+\left(\xi_{t}+a \xi_{x}\right) u_{\xi}=0, \quad 0 \leq \xi \leq 1, \quad \tau \geq 0,
$$

where the transformation relation is given by

$$
\xi(x, t)=\frac{x-x_{0}(t)}{x_{1}(t)-x_{0}(t)} .
$$

For simplicity, equation (3) is multiplied with $\xi_{x}^{-1}$,

$$
\left(\xi_{x}\right)^{-1} u_{t}+\left[a+a^{(\xi)}\right] u_{\xi}=0, \quad 0 \leq \xi \leq 1, \quad t \geq 0
$$

which is a variable coefficient partial differential equation with

$$
a^{(\xi)}(\xi, t)=\frac{\xi_{t}}{\xi_{x}}=\xi\left[\dot{x}_{0}(t)-\dot{x}_{1}(t)\right] .
$$

The energy method (multiply with the solution and integrate over the domain) applied to (5) leads to

$$
\frac{d}{d t}\|u\|_{x_{1}-x_{0}}^{2}=\left(a-\dot{x}_{0}\right) u(0, t)^{2}-\left(a-\dot{x}_{1}\right) u(1, t)^{2},
$$

where the norm is defined by $\|u\|_{x_{1}-x_{0}}^{2}=\int_{0}^{1}\left(x_{1}(t)-x_{0}(t)\right) u^{2} d \xi$. In order to bound the energy of the solution by data, the boundary conditions are given by

$$
\begin{aligned}
& u(0, t)=g_{0}(t), \text { if } a-\dot{x}_{0}>0 \\
& u(1, t)=g_{1}(t), \text { if } a-\dot{x}_{1}<0,
\end{aligned}
$$

which leads to

$$
\begin{aligned}
& \frac{d}{d t}\|u\|_{x_{1}-x_{0}}^{2}=\left(a-\dot{x}_{0}\right) g_{0}(t)^{2}-\left(a-\dot{x}_{1}\right) u(1, t)^{2}, \quad \text { if } a-\dot{x}_{0}>0, a-\dot{x}_{1} \geq 0 \\
& \frac{d}{d t}\|u\|_{x_{1}-x_{0}}^{2}=\left(a-\dot{x}_{0}\right) g_{0}(t)^{2}-\left(a-\dot{x}_{1}\right) g_{1}(t)^{2}, \quad \text { if } a-\dot{x}_{0}>0, a-\dot{x}_{1}<0 \\
& \frac{d}{d t}\|u\|_{x_{1}-x_{0}}^{2}=\left(a-\dot{x}_{0}\right) u(0, t)^{2}-\left(a-\dot{x}_{1}\right) g_{1}(t)^{2}, \quad \text { if } a-\dot{x}_{0} \leq 0, a-\dot{x}_{1}<0 \\
& \frac{d}{d t}\|u\|_{x_{1}-x_{0}}^{2}=\left(a-\dot{x}_{0}\right) u(0, t)^{2}-\left(a-\dot{x}_{1}\right) u(1, t)^{2}, \text { if } a-\dot{x}_{0} \leq 0, a-\dot{x}_{1} \geq 0
\end{aligned}
$$

\section{B. The two dimensional problem}

Consider the following two dimensional advection equation in a moving domain

$$
u_{t}+a u_{x}+b u_{y}=0, \quad(x, y) \in \Omega(t), \quad t \geq 0,
$$

where $a$ and $b$ are constant wave speeds in the $x$ and $y$ directions respectively. A transformation from the moving domain into a fixed domain such that $0 \leq \xi \leq 1$ and $0 \leq \eta \leq 1$, and $\tau=t$ is given by

$$
\begin{array}{ll}
x=x(\xi, \eta, \tau), & y=y(\xi, \eta, \tau) \\
\xi=\xi(x, y, t), & \eta=\eta(x, y, t) .
\end{array}
$$


The chain-rule is employed to interpret the differential equation (10) in terms of the fixed coordinates as

$$
u_{\tau}+\left(\xi_{t}+a \xi_{x}+b \xi_{y}\right) u_{\xi}+\left(\eta_{t}+a \eta_{x}+b \eta_{y}\right) u_{\eta}=0, \quad 0 \leq \xi \leq 1, \quad 0 \leq \eta \leq 1, \quad \tau \geq 0 .
$$

The Jacobian matrix of the transformation, $[J]$, that maps the derivatives of the solution with respect to the moving coordinates $\left(u_{x}, u_{y}, u_{t}\right)^{T}$ to those of the corresponding fixed coordinates $\left(u_{\xi}, u_{\eta}, u_{\tau}\right)^{T}$, is

$$
[J]=\left(\begin{array}{lll}
x_{\xi} & y_{\xi} & 0 \\
x_{\eta} & y_{\eta} & 0 \\
x_{\tau} & y_{\tau} & 1
\end{array}\right)
$$

The relation between $[J]$, and its inverse, which transforms the derivatives back to the moving coordinates leads to the metric relations

$$
\begin{array}{lll}
\xi_{t}=\frac{x_{\eta} y_{\tau}-x_{\tau} y_{\eta}}{J}, & \xi_{x}=\frac{y_{\eta}}{J}, & \xi_{y}=\frac{-x_{\eta}}{J} \\
\eta_{t}=\frac{y_{\xi} x_{\tau}-x_{\xi} y_{\tau}}{J}, & \eta_{x}=\frac{-y_{\xi}}{J}, & \eta_{y}=\frac{x_{\xi}}{J}
\end{array}
$$

in which $J>0$ is the determinant of $[J]$. With the aid of (14) the coefficients in (12) are replaced in terms of the derivatives of the moving coordinates. The transformed differential equation in the fixed domain becomes

$$
J u_{\tau}+[\alpha+\tilde{\alpha}] u_{\xi}+[\beta+\tilde{\beta}] u_{\eta}=0, \quad 0 \leq \xi \leq 1, \quad 0 \leq \eta \leq 1 \quad \tau \geq 0 .
$$

Equation (15) is a variable coefficient partial differential equation where $\alpha=x_{\eta} y_{\tau}-x_{\tau} y_{\eta}, \tilde{\alpha}=a y_{\eta}-b x_{\eta}$, $\beta=y_{\xi} x_{\tau}-x_{\xi} y_{\tau}, \tilde{\beta}=-a y_{\xi}+b x_{\xi}$, and $J=x_{\xi} y_{\eta}-x_{\eta} y_{\xi}$. Note the similarity with (5).

The energy method (multiply with the solution and integrate over the domain) applied to (15) leads to

$$
\frac{d}{d \tau}\|u\|_{J}^{2}=-\oint u^{2}(\hat{\alpha}, \hat{\beta}) \cdot n d s
$$

where the norm is defined by $\|u\|_{J}^{2}=\iint u^{2} J d \xi d \eta$, in the fixed domain $\Omega$. Moreover $\hat{\alpha}=\alpha+\tilde{\alpha}$ and $\hat{\beta}=\beta+\tilde{\beta}, n$ is the unit normal vector pointing outward from $\Omega$, and $d s$ is an infinitesimal element along the boundary of $\Omega$. A schematic of the physical and computational domain, and boundary definition is presented in Figure 1. In order to bound the energy of the solution by data, boundary conditions must be applied

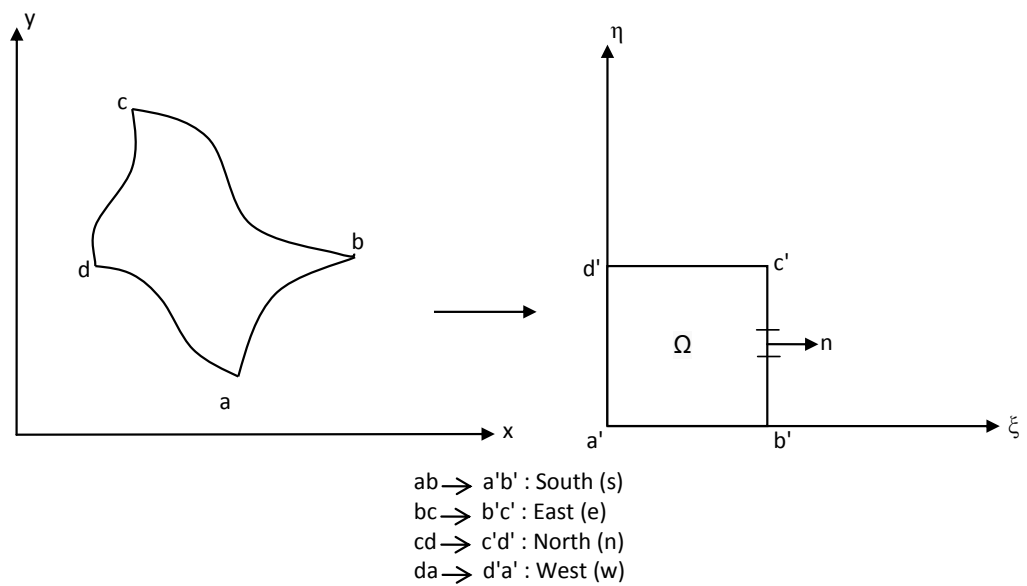

Figure 1. A schematic of the physical and computational domains and boundary definitions.

when $(\hat{\alpha}, \hat{\beta}) \cdot n<0$, which defines an inflow condition. The energy rate in (16) is bounded by evaluating $(\hat{\alpha}, \hat{\beta}) \cdot n$ along each boundary of the computational domain, denoted by $s, w, n, e$, and imposing boundary conditions defined by 


$$
\begin{aligned}
& u_{s}(\xi, 0, t)=g_{s}(\xi, t) \quad \text { if } \quad(\hat{\alpha}, \hat{\beta}) \cdot n=-\hat{\beta}_{s}<0 \\
& u_{n}(\xi, 1, t)=g_{n}(\xi, t) \quad \text { if } \quad(\hat{\alpha}, \hat{\beta}) \cdot n=\hat{\beta}_{n}<0 \\
& u_{w}(0, \eta, t)=g_{w}(\eta, t) \quad \text { if } \quad(\hat{\alpha}, \hat{\beta}) \cdot n=-\hat{\alpha}_{w}<0 \\
& u_{e}(1, \eta, t)=g_{e}(\eta, t) \quad \text { if } \quad(\hat{\alpha}, \hat{\beta}) \cdot n=\hat{\alpha}_{e}<0 .
\end{aligned}
$$

The continuous energy estimate, using (17) is obtained as

$$
\frac{d}{d \tau}\|u\|_{J}^{2}=-\oint\left(\begin{array}{c}
\hat{u}_{s} \\
\hat{u}_{n} \\
\hat{u}_{w} \\
\hat{u}_{e}
\end{array}\right)^{T}\left(\begin{array}{cccc}
-\hat{\beta}_{s} & & & \\
& \hat{\beta}_{n} & & \\
& & -\hat{\alpha}_{w} & \\
& & & \hat{\alpha}_{e}
\end{array}\right)\left(\begin{array}{c}
\hat{u}_{s} \\
\hat{u}_{n} \\
\hat{u}_{w} \\
\hat{u}_{e}
\end{array}\right) d s .
$$

In (18) we have $\hat{u}_{k}=g_{k}$ if we are at an inflow boundary and a boundary condition is required. At an outflow we have $\hat{u}_{l}=u_{l}$ and no boundary condition is required.

\section{The semi-discrete problem}

\section{A. The one dimensional problem}

The numerical solution is given by $v=\left[v_{0}, v_{1}, \ldots, v_{N}\right]^{T}$, where $v_{i}(t)$ approximates $u\left(x_{i}, t\right)$. The first order derivative $u_{x}$ is approximated by $D v$, where $D$ is a so called summation-by-parts (SBP) operator of the form

$$
D=P^{-1} Q .
$$

$P$ is a positive definite symmetric matrix, and $Q$ is an almost skew-symmetric matrix that satisfies

$$
Q+Q^{T}=E_{N}-E_{0}=B=\operatorname{diag}(-1,0, \ldots, 0,1) .
$$

In $(20), E_{0}=\operatorname{diag}(1,0, \ldots, 0)$ and $E_{N}=\operatorname{diag}(0, \ldots, 0,1)$. We define the scalar product by $(w, v)=w^{T} P v$ and the discrete norm as

$$
\|v\|_{P}^{2}=v^{T} P v .
$$

A first derivative SBP operator is a 2s-order accurate central difference operator which is modified close to the boundaries such that it becomes one-sided. Together with the diagonal norm, the boundary closure is s-order accurate, making the SBP operator $\mathrm{s}+1$ order accurate globally. ${ }^{5}$

To obtain an energy estimate where the growth rate of the numerical solution correspond to the continuous one, the splitting technique ${ }^{4}$ must be used. An equivalent form of (5) is

$$
\left(\xi_{x}\right)^{-1} u_{t}+\frac{1}{2}\left[\left(a+a^{(\xi)}\right) u\right]_{\xi}-\frac{1}{2} a_{\xi}^{(\xi)} u+\frac{1}{2}\left[a+a^{(\xi)}\right] u_{\xi}=0, \quad 0 \leq \xi \leq 1, \quad t \geq 0 .
$$

The SBP-SAT approximation of (22) including the weak form of the boundary conditions is now obtained as

$$
\begin{aligned}
\bar{J} v_{t}+\frac{1}{2} P^{-1} Q\left[A^{(\xi)}+a I\right] v-\frac{1}{2} A_{\xi}^{(\xi)} v+\frac{1}{2}\left[A^{(\xi)}+a I\right] P^{-1} Q v= & \sigma_{0}(t) P^{-1}\left[E_{0} v-g_{0}(t) e_{0}\right]+ \\
& \sigma_{1}(t) P^{-1}\left[E_{N} v-g_{1}(t) e_{N}\right],
\end{aligned}
$$

where $\bar{J}$ is a diagonal matrix having the numerical values of $\xi_{x}^{-1}$ on the main diagonal, and I is the indentity matrix (the dimensions are consistent with the grid). The vectors $e_{0}$ and $e_{N}$ have zero entries except for one element which is equal to one at the first and last positions respectively. The coefficients $\sigma_{0}(t)$ and $\sigma_{1}(t)$ (which enforce the boundary conditions) will be determined by stability requirements. Moreover $A^{(\xi)}$ is a diagonal matrix with the numerical values of $a^{(\xi)}$ on the main diagonal.

The energy method (multiply from the left with $v^{T} P$ ) applied to (23) leads to

$$
\begin{aligned}
v^{T} P \bar{J} v_{t}+\frac{1}{2} v^{T}\left[Q\left(A^{(\xi)}+a I\right)+\left(A^{(\xi)}+a I\right) Q\right] v-\frac{1}{2} v^{T} A_{\xi}^{(\xi)} v= & \sigma_{0}(t) v_{0}\left[v_{0}-g_{0}(t)\right]+ \\
& \sigma_{1}(t) v_{N}\left[v_{N}-g_{1}(t)\right] .
\end{aligned}
$$


Let $Q=\frac{Q+Q^{T}}{2}+\frac{Q-Q^{T}}{2}$ and use $(20)$ in $(24)$, to get

$$
v^{T} P \bar{J} v_{t}-\frac{1}{2} v^{T} A_{\xi}^{(\xi)} v+\frac{1}{2} v^{T}\left[B\left(A^{(\xi)}+a I\right)\right] v=\sigma_{0}(t) v_{0}\left[v_{0}-g_{0}(t)\right]+\sigma_{1}(t) v_{N}\left[v_{N}-g_{1}(t)\right] .
$$

Note that $A_{\xi}^{(\xi)}$ has the numerical values of $-\bar{J}_{t}$ on the main diagonal which leads to

$$
\frac{1}{2} \frac{d}{d t}\|v\|_{P \bar{J}}^{2}=-\frac{1}{2}\left[\left(a^{(\xi)}+a\right)\right]_{N} v_{N}^{2}+\frac{1}{2}\left[\left(a^{(\xi)}+a\right)\right]_{0} v_{0}^{2}+\sigma_{0}(t) v_{0}\left[v_{0}-g_{0}(t)\right]+\sigma_{1}(t) v_{N}\left[v_{N}-g_{1}(t)\right] .
$$

By rearranging we get the final result

$$
\frac{d}{d t}\|v\|_{P\left(x_{1}-x_{0}\right)}^{2}=\left(a-\dot{x}_{0}\right) v_{0}^{2}-\left(a-\dot{x}_{1}\right) v_{N}^{2}+2 \sigma_{0}(t) v_{0}\left[v_{0}-g_{0}(t)\right]+2 \sigma_{1}(t) v_{N}\left[v_{N}-g_{1}(t)\right],
$$

in which the norm is time-dependent, similar to the continous norm and defined by $\|v\|_{P\left(x_{1}-x_{0}\right)}^{2}=v^{T} P \bar{J} v$.

In order to have a stable scheme the penalty coefficients are defined as

$$
\sigma_{0}=-\frac{\left(a-\dot{x}_{0}\right)+\left|a-\dot{x}_{0}\right|}{2}, \quad \sigma_{1}=\frac{\left(a-\dot{x}_{1}\right)-\left|a-\dot{x}_{1}\right|}{2},
$$

which leads to the energy estimates

$$
\begin{array}{lrl}
\frac{d}{d t}\|v\|_{P\left(x_{1}-x_{0}\right)}^{2}=\left(a-\dot{x}_{0}\right) g_{0}(t)^{2}-\left(a-\dot{x}_{1}\right) v_{N}^{2}-\left(a-\dot{x}_{0}\right)\left[v_{0}-g_{0}(t)\right]^{2}, & \text { if } a-\dot{x}_{0}>0, a-\dot{x}_{1} \geq 0, \\
\frac{d}{d t}\|v\|_{P\left(x_{1}-x_{0}\right)}^{2}=\left(a-\dot{x}_{0}\right) g_{0}(t)^{2}-\left(a-\dot{x}_{1}\right) g_{1}(t)^{2}-\left(a-\dot{x}_{0}\right)\left[v_{0}-g_{0}(t)\right]^{2}+\left(a-\dot{x}_{1}\right)\left[v_{N}-g_{1}(t)\right]^{2}, & & \\
\frac{d}{d t}\|v\|_{P\left(x_{1}-x_{0}\right)}^{2}=\left(a-\dot{x}_{0}\right) v_{0}^{2}-\left(a-\dot{x}_{1}\right) g_{1}(t)^{2}+\left(a-\dot{x}_{1}\right)\left[v_{N}-g_{1}(t)\right]^{2}, & \text { if } a-\dot{x}_{0}>0, a-\dot{x}_{1}<0, a-\dot{x}_{1}<0, \\
\frac{d}{d t}\|v\|_{P\left(x_{1}-x_{0}\right)}^{2}=\left(a-\dot{x}_{0}\right) v_{0}^{2}-\left(a-\dot{x}_{1}\right) v_{N}^{2}, & \text { if } a-\dot{x}_{0} \leq 0, a-\dot{x}_{1} \geq 0 .
\end{array}
$$

Note that the stability analysis above is independent of the order of accuracy of SBP operators. Note also that (29) is similar to (9) with added damping terms proportional to the error at the boundaries.

\section{B. The two dimensional problem}

The computational domain $\Omega$ in the two dimensional case is a square in $\xi, \eta$ coordinates, see Figure 1 , and discretized using $N$ and $M$ nodes in the direction of $\xi$ and $\eta$ respectively. The numerical solution is arranged as a column vector of size $N \times M$ where $V(t)=\left[V_{0}(t), V_{1}(t), \ldots, V_{N}(t)\right]^{T} . V_{i}(t)$ is a column vector of size $M$ and approximates the solution $u\left(\xi_{i}, \eta, \tau\right)$ by its numerical values at the corresponding grids in the $\eta$ direction. A finite difference operator for a two dimensional field is constructed by extending the one dimensional SBP operators written as

$$
D_{\xi}=P_{\xi}^{-1} Q_{\xi} \otimes I_{\eta}, D_{\eta}=I_{\xi} \otimes P_{\eta}^{-1} Q_{\eta},
$$

where $\otimes$ represents the Kronecker product. The first order derivatives $u_{\xi}$ and $u_{\eta}$ are approximated by finite difference matrices $D_{\xi} V$ and $D_{\eta} V$ respectively. Note that all matrices in the first position are of size $N \times N$, and the second position $M \times M$. I denotes the identity matrix of a size consistent with its position in the Kronecker product.

The corresponding discrete norm is introduced as

$$
\|V\|_{P_{\xi} \otimes P_{\eta}}^{2}=V^{T}\left(P_{\xi} \otimes P_{\eta}\right) V .
$$

To obtain an energy estimate where the growth rate corresponds to the continuous one, we again need to use the splitting technique ${ }^{4}$ as in the one-dimensional case. We split (12) as

$$
\begin{aligned}
J u_{\tau} & +\frac{1}{2}[(\alpha+\tilde{\alpha}) u]_{\xi}+\frac{1}{2}[\alpha+\tilde{\alpha}] u_{\xi}-\frac{1}{2}[\alpha+\tilde{\alpha}]_{\xi} u \\
& +\frac{1}{2}[(\beta+\tilde{\beta}) u]_{\eta}+\frac{1}{2}[\beta+\tilde{\beta}] u_{\eta}-\frac{1}{2}[\beta+\tilde{\beta}]_{\eta} u=0 .
\end{aligned}
$$


The SBP-SAT approximation of (32) including the penalty terms for the weak boundary conditions at the different boundaries, see Figure 1, is constructed as

$$
\begin{array}{rllll}
\bar{J} V_{t} & +\frac{1}{2}\left(P_{\xi}^{-1} Q_{\xi} \otimes I\right)[A+\tilde{A}] V & +\frac{1}{2}[A+\tilde{A}]\left(P_{\xi}^{-1} Q_{\xi} \otimes I\right) V & - & \frac{1}{2}[A+\tilde{A}]_{\xi} V \\
& +\frac{1}{2}\left(I \otimes P_{\eta}^{-1} Q_{\eta}\right)[B+\tilde{B}] V & +\frac{1}{2}[B+\tilde{B}]\left(I \otimes P_{\eta}^{-1} Q_{\eta}\right) V & - & \frac{1}{2}[B+\tilde{B}]_{\eta} V \\
= & \left(\Sigma_{s} \otimes E_{0}\right)\left(P_{\xi}^{-1} \otimes P_{\eta}^{-1}\right)\left(V-G_{s}\right) & +\left(E_{0} \otimes \Sigma_{w}\right)\left(P_{\xi}^{-1} \otimes P_{\eta}^{-1}\right)\left(V-G_{w}\right) & + \\
& \left(\Sigma_{n} \otimes E_{1}\right)\left(P_{\xi}^{-1} \otimes P_{\eta}^{-1}\right)\left(V-G_{n}\right) & + & \left(E_{1} \otimes \Sigma_{e}\right)\left(P_{\xi}^{-1} \otimes P_{\eta}^{-1}\right)\left(V-G_{e}\right) . &
\end{array}
$$

In (33), $\bar{J}, A, \tilde{A}, B$ and $\tilde{B}$ are diagonal matrices of size $N M \times N M$ which approximate $J, \alpha, \tilde{\alpha}, \beta$ and $\tilde{\beta}$ respectively. $E_{0}$ and $E_{1}$ are zero matrices except for the first and last entry which is one. The penalty matrices $\Sigma_{s}, \Sigma_{e}, \Sigma_{w}$ and $\Sigma_{n}$ at the corresponding boundaries will be determined by stability requirements.

The energy method (multiply (33) from the left with $\left.V^{T}\left(P_{\xi} \otimes P_{\eta}\right)\right)$ leads to

$$
\begin{aligned}
V^{T}\left(P_{\xi} \otimes P_{\eta}\right) \bar{J} V_{\tau} & +\frac{1}{2} V^{T}\left(Q_{\xi} \otimes P_{\eta}\right) \hat{A} V+\frac{1}{2} V^{T} \hat{A}\left(Q_{\xi} \otimes P_{\eta}\right) V-\frac{1}{2} V^{T}\left(P_{\xi} \otimes P_{\eta}\right) \hat{A}_{\xi} V \\
& +\frac{1}{2} V^{T}\left(P_{\xi} \otimes Q_{\eta}\right) \hat{B} V+\frac{1}{2} V^{T} \hat{B}\left(P_{\xi} \otimes Q_{\eta}\right) V-\frac{1}{2} V^{T}\left(P_{\xi} \otimes P_{\eta}\right) \hat{B}_{\eta} V \\
& =V^{T}\left(\Sigma_{s} \otimes E_{0}\right)\left(I_{\xi} \otimes I_{\eta}\right)(V-G s)+V^{T}\left(E_{0} \otimes \Sigma_{w}\right)\left(I_{\xi} \otimes I_{\eta}\right)(V-G w) \\
& +V^{T}\left(\Sigma_{n} \otimes E_{1}\right)\left(I_{\xi} \otimes I_{\eta}\right)(V-G n)+V^{T}\left(\Sigma_{e} \otimes E_{1}\right)\left(I_{\xi} \otimes I_{\eta}\right)(V-G e),
\end{aligned}
$$

where $\hat{A}=A+\tilde{A}$, and $\hat{B}=B+\tilde{B}$. Note that in order to arrive at (34), we have used that the diagonal matrix $P_{\xi} \otimes P_{\eta}$ commute with $\hat{A}$ and $\hat{B}$. The properties of the Kronecker products leads to

$$
\begin{aligned}
V^{T}\left(P_{\xi} \otimes P_{\eta}\right) \bar{J} V_{\tau} & +V_{\tau}^{T} \bar{J}\left(P_{\xi} \otimes P_{\eta}\right) V-V^{T}\left(P_{\xi} \otimes P_{\eta}\right)\left(\hat{A}_{\xi}+\hat{B}_{\eta}\right) V= \\
& -\frac{1}{2} V^{T}\left[\left(Q_{\xi}+Q_{\xi}^{T}\right) \otimes P_{\eta}\right] \hat{A} V-\frac{1}{2} V^{T} \hat{A}\left[\left(Q_{\xi}+Q_{\xi}^{T}\right) \otimes P_{\eta}\right] V \\
& -\frac{1}{2} V^{T}\left[P_{\xi} \otimes\left(Q_{\eta}+Q_{\eta}^{T}\right)\right] \hat{B} V-\frac{1}{2} V^{T} \hat{B}\left[P_{\xi} \otimes\left(Q_{\eta}+Q_{\eta}^{T}\right)\right] V \\
& +2 V^{T}\left(\Sigma_{s} \otimes E_{0}\right)\left(I_{\xi} \otimes I_{\eta}\right)(V-G s)+2 V^{T}\left(E_{0} \otimes \Sigma_{w}\right)\left(I_{\xi} \otimes I_{\eta}\right)(V-G w) \\
& +2 V^{T}\left(\Sigma_{n} \otimes E_{1}\right)\left(I_{\xi} \otimes I_{\eta}\right)(V-G n)+2 V^{T}\left(\Sigma_{e} \otimes E_{1}\right)\left(I_{\xi} \otimes I_{\eta}\right)(V-G e) .
\end{aligned}
$$

If we ignore the data in (35) and simplify it by using the SBP property (20), we obtain

$$
\begin{aligned}
& V^{T}\left(P_{\xi} \otimes P_{\eta}\right) \bar{J} V_{\tau}+V_{\tau}^{T} \bar{J}\left(P_{\xi} \otimes P_{\eta}\right) V-V^{T}\left(P_{\xi} \otimes P_{\eta}\right)\left(\hat{A}_{\xi}+\hat{B}_{\eta}\right) V= \\
& \left(\begin{array}{c}
V_{w} \\
V_{s} \\
V_{E} \\
V_{n}
\end{array}\right)\left(\begin{array}{cccc}
\hat{A}_{w}+2 \Sigma_{w} & & & \\
& \hat{B}_{s}+2 \Sigma_{s} & & \\
& & -\hat{A}_{e}+2 \Sigma_{e} & \\
& & -\hat{B}_{n}+2 \Sigma_{n}
\end{array}\right)\left(\begin{array}{cccc}
P_{\eta} & & & \\
& P_{\xi} & & \\
& & P_{\eta} & \\
& & & P_{\xi}
\end{array}\right)\left(\begin{array}{c}
V_{w} \\
V_{s} \\
V_{E} \\
V_{n}
\end{array}\right) .
\end{aligned}
$$

To form the time-derivative of the norm of the numerical solution similar to the continuous one, $\bar{J}, \hat{A}_{\xi}$ and $\hat{B}_{\eta}$ must be defined such that

$$
\hat{A}_{\xi}+\hat{B}_{\eta}=-\bar{J}_{\tau}
$$

The relation (37) transforms the left hand side of (36) into $\frac{d}{d t}\|V\|_{\left(P_{\xi} \otimes P_{\eta}\right) \bar{J}}^{2}$. Note that the $\hat{A}=A+\tilde{A}$ and $\hat{B}=B+\tilde{B}$ are the numerical values for $J \xi_{t}+J\left(a \xi_{x}+b \xi_{y}\right)$ and $J \eta_{t}+J\left(a \eta_{x}+b \eta_{y}\right)$ respectively in (37). If we expand (37) using these relations we find that the following equation must hold,

$$
\bar{J}_{\tau}+\left(\overline{J \xi}_{t}\right)_{\xi}+\left(\bar{J}_{t}\right)_{\eta}+a\left[\left(J \bar{\xi}_{x}\right)_{\xi}+\left(\bar{J}_{x}\right)_{\eta}\right]+b\left[\left(J \bar{\xi}_{y}\right)_{\xi}+\left(\bar{J}_{y}\right)_{\eta}\right]=0 .
$$

The bar signs in (38) should bring the numerical evaluations of the expressions in mind. Relation (38) is satisfied if the so-called Geometric Conservation Law (GCL) $)^{2,3}$ holds. The GCL is summerized as

$$
J_{\tau}+\left(J \xi_{t}\right)_{\xi}+\left(J \eta_{t}\right)_{\eta}=0, \quad\left(J \xi_{x}\right)_{\xi}+\left(J \eta_{x}\right)_{\eta}=0, \quad\left(J \xi_{y}\right)_{\xi}+\left(J \eta_{y}\right)_{\eta}=0,
$$

where the first relation in (39) conserves the volume and the other two conserve the surface ${ }^{3}$ on a moving and/or deforming domain. Consider the volume conservation relation as an example. Both the Jacobian and time metrics can be defined equivalently in the so-called asymmetric conservative form $^{3}$ as 


$$
\bar{J}=\left(M^{1}\right)_{\eta}-\left(M^{2}\right)_{\xi}, \quad A=\left(M^{2}\right)_{\tau}-\left(M^{3}\right)_{\eta}, \quad B=\left(M^{3}\right)_{\xi}-\left(M^{1}\right)_{\tau},
$$

where $M^{1}=x_{\xi} y, M^{2}=x_{\eta} y, M^{3}=x_{\tau} y$. Substituting (40) into the volume conservation part of (39) leads to

$$
\left[\left(M^{1}\right)_{\eta}-\left(M^{2}\right)_{\xi}\right]_{\tau}+\left[\left(M^{2}\right)_{\tau}-\left(M^{3}\right)_{\eta}\right]_{\xi}+\left[\left(M^{3}\right)_{\xi}-\left(M^{1}\right)_{\tau}\right]_{\eta}=0 .
$$

The relation (41) gives us a clear condition on the finite difference operators such that the volume conservation law is numerically fulfilled.

Assume now that time is continuous. Equation (41) is fulfilled analytically. If a unique difference operator is used for each direction in the transformation relations and the numerical scheme, the volume is conserved also in the discrete case since (41) goes to

$$
\left[\left(I_{\xi} \otimes D_{\eta}\right) M^{1}\right]_{\tau}-\left(I_{\xi} \otimes D_{\eta}\right)\left(M^{1}\right)_{\tau}-\left[\left(D_{\xi} \otimes I_{\eta}\right) M^{2}\right]_{\tau}+\left(D_{\xi} \otimes I_{\eta}\right)\left(M^{2}\right)_{\tau}-\left(D_{\xi} \otimes D_{\eta}\right) M^{3}+\left(D_{\xi} \otimes D_{\eta}\right) M^{3}=0 .
$$

With an analogous strategy, one can prove that the surface relations also hold and subsequently both the GCL and condition (37) is satisfied in the discrete case.

The norm in the discrete case is time-dependent, similar to the continuous norm and defined by $\|V\|_{\bar{J}\left(P_{\xi} \otimes P_{\eta}\right)}^{2}=$ $V^{T} \bar{J}\left(P_{\xi} \otimes P_{\eta}\right) V$. In order to have a stable scheme, the time derivative of the solution norm should not grow, meaning that the right hand side of (36) must be negative semi-definite. Since $P>0$ we require

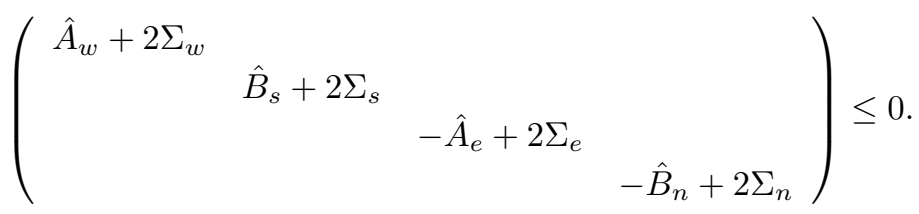

For (43) to be fulfiled, $\Sigma_{w}, \Sigma_{s}, \Sigma_{e}$ and $\Sigma_{n}$ are chosen such that

$$
\begin{aligned}
& \Sigma_{w} \leq \frac{-\hat{A}_{w}}{2} \quad \text { if } \hat{A}_{w}>0 \text { and } \Sigma_{w}=0 \text { if } \hat{A}_{w} \leq 0 \\
& \Sigma_{s} \leq \frac{-\hat{B}_{s}}{2} \text { if } \hat{B}_{s}>0 \text { and } \Sigma_{s}=0 \text { if } \hat{B}_{s} \leq 0 \\
& \Sigma_{e} \leq \frac{\hat{A}_{e}}{2} \text { if } \hat{A}_{e}<0 \text { and } \Sigma_{e}=0 \text { if } \hat{A}_{e} \geq 0 \\
& \Sigma_{n} \leq \frac{\hat{A_{n}}}{2} \quad \text { if } \hat{B}_{n}<0 \text { and } \Sigma_{n}=0 \text { if } \hat{B}_{n} \leq 0 \text {. }
\end{aligned}
$$

A general formulation that automatically defines the penalty coefficients in an appropriate way is

$$
\Sigma_{w}=-\frac{\hat{A}_{w}+\left|\hat{A}_{w}\right|}{2}, \quad \Sigma_{s}=-\frac{\hat{B}_{s}+\left|\hat{B}_{s}\right|}{2}, \quad \Sigma_{e}=\frac{\hat{A}_{e}-\left|\hat{A}_{e}\right|}{2}, \quad \Sigma_{n}=\frac{\hat{B}_{n}-\left|\hat{B}_{n}\right|}{2} .
$$

The numerical energy estimate, including the boundary procedures can now be formulated as

$$
\begin{aligned}
& \frac{d}{d \tau}\|V\|_{\bar{J}\left(P_{\xi} \otimes P_{\eta}\right)}^{2}= \\
& \left(\begin{array}{c}
V_{s} \\
V_{n} \\
V_{w} \\
V_{e}
\end{array}\right)^{T}\left(\begin{array}{cccc}
\left(\hat{B}_{s}+2 \Sigma_{s}\right) P_{\xi} & & & \\
& \left(-\hat{B}_{n}+2 \Sigma_{n}\right) P_{\xi} & & \\
& & \left(\hat{A}_{w}+2 \Sigma_{w}\right) P_{\eta} & \\
& & \left(-\hat{A}_{e}+2 \Sigma_{e}\right) P_{\eta}
\end{array}\right)\left(\begin{array}{c}
V_{s} \\
V_{n} \\
V_{w} \\
V_{e}
\end{array}\right)- \\
& \left(\begin{array}{c}
V_{s} \\
V_{n} \\
V_{w} \\
V_{e}
\end{array}\right)^{T}\left(\begin{array}{cccc}
2 \Sigma_{s} P_{\xi} & & & \\
& 2 \Sigma_{n} P_{\xi} & & \\
& & 2 \Sigma_{w} P_{\eta} & \\
& & & 2 \Sigma_{e} P_{\eta}
\end{array}\right)\left(\begin{array}{c}
\hat{V}_{s} \\
\hat{V}_{n} \\
\hat{V}_{w} \\
\hat{V}_{e}
\end{array}\right)
\end{aligned}
$$


where $\hat{V}_{i}=G_{i}$ if the boundary procedure is required, and $\hat{V}_{i}=V_{i}$ if no boundary procedure is required, $i \in\{s, n, w, e\}$. For illustration, consider the south boundary and the corresponding numerical contribution to the energy estimate if a boundary condition is necessary. It becomes

$$
\frac{d}{d \tau}\|V\|_{\bar{J}_{s}\left(P_{\xi} \otimes P_{\eta}\right)}^{2}=G_{s}^{T} \hat{B}_{s} P_{\xi} G_{s}-\left(V_{s}-G_{s}\right)^{T} \hat{B}_{s} P_{\xi}\left(V_{s}-G_{s}\right)+B T,
$$

where $B T$ denotes the contribution from the other boundaries. Equation (47) shows that the numerical energy estimate is similar to the continous one in (18) and that an extra damping term is added. The result (47) is similar to the one-dimensional result in (29).

\section{Numerical experiments}

\section{A. The one dimensional problem}

In our one-dimensional example we move the boundaries using

$$
x_{0}(t)=-\frac{1}{\pi} \sin (2 \pi t), x_{1}(t)=1-\frac{2}{3 \pi} \sin (3 \pi t) .
$$

A schematic of the moving grids in the $x, t$ system (red curves) and the corresponding fixed grids in the $\xi, t$ system (black lines) are presented in Figure 2. This schematic is for illustration purposes and the numerical experiments are carried out on finer meshes with 161 grid points on the finest case.

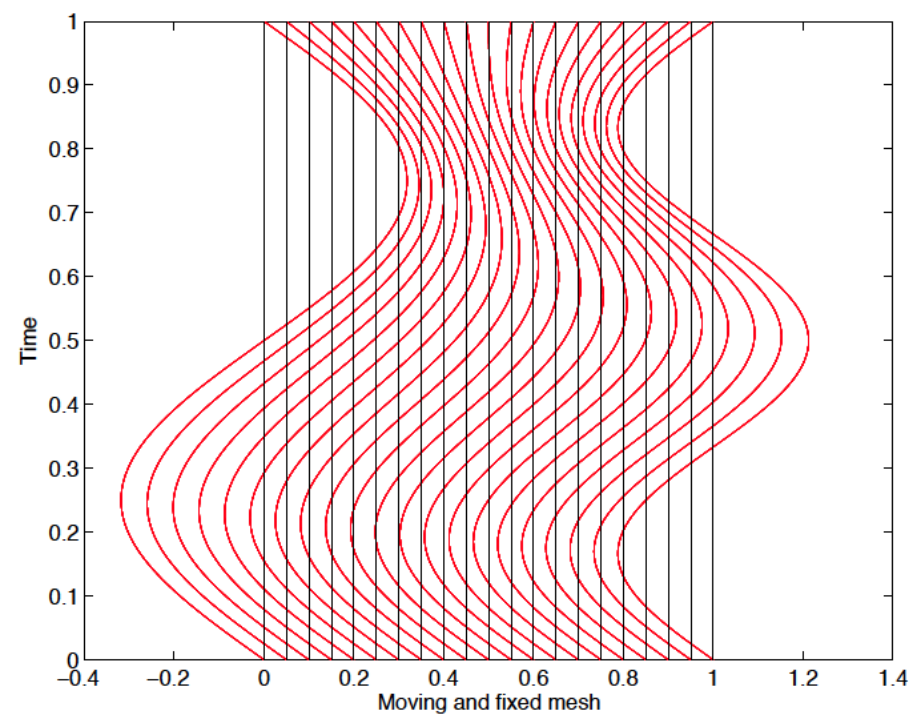

Figure 2. A schematic of a moving and fixed mesh in $x$ and $\xi$ coordinates respectively.

In all the experiments we used $a=1$. The penalty coefficients corresponding to each boundary, leading to a stable scheme are schematically presented in Figure 3 . When the penalty coefficient is zero, the corresponding boundary procedure is not activated at that boundary (one time interval marked with No B.C. in Figure 3. Moreover based on the speed of the boundaries, there is possibility for either having both inflow and outflow boundary procedures simultaneously (two time intervals marked with 2 B.C's in Figure 3 or having only one boundary procedure (three time intervals marked by 1 B.C. in Figure 3 during computations.

The convergence rate is calculated as

$$
p=\frac{\log _{10}\left(\frac{\left\|u-v^{(1)}\right\|_{P}}{\left\|u-v^{(2)}\right\|_{P}}\right)}{\log _{10}\left(\frac{N^{(1)}}{N^{(2)}}\right)}
$$




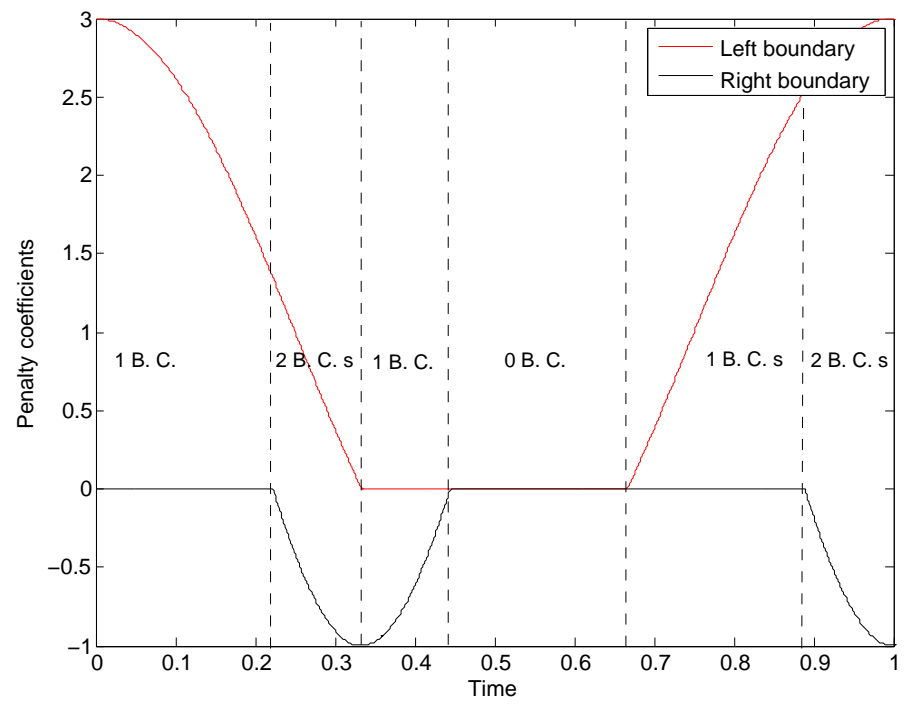

Figure 3. Penalty coefficients versus time for left and right boundaries in the one-dimensional example.

in which $u$ is the exact solution, $v^{(1)}$ and $v^{(2)}$ are the corresponding numerical solutions on the meshes of $N^{(1)}$ and $N^{(2)}$ grid points (including boundary points) respectively.

The numerical solution in our experiments converges to the exact solution at $\mathrm{t}=1$ with the convergence rates presented in Figure 4. The convergence rates are in agreement with the theory mentioned above.

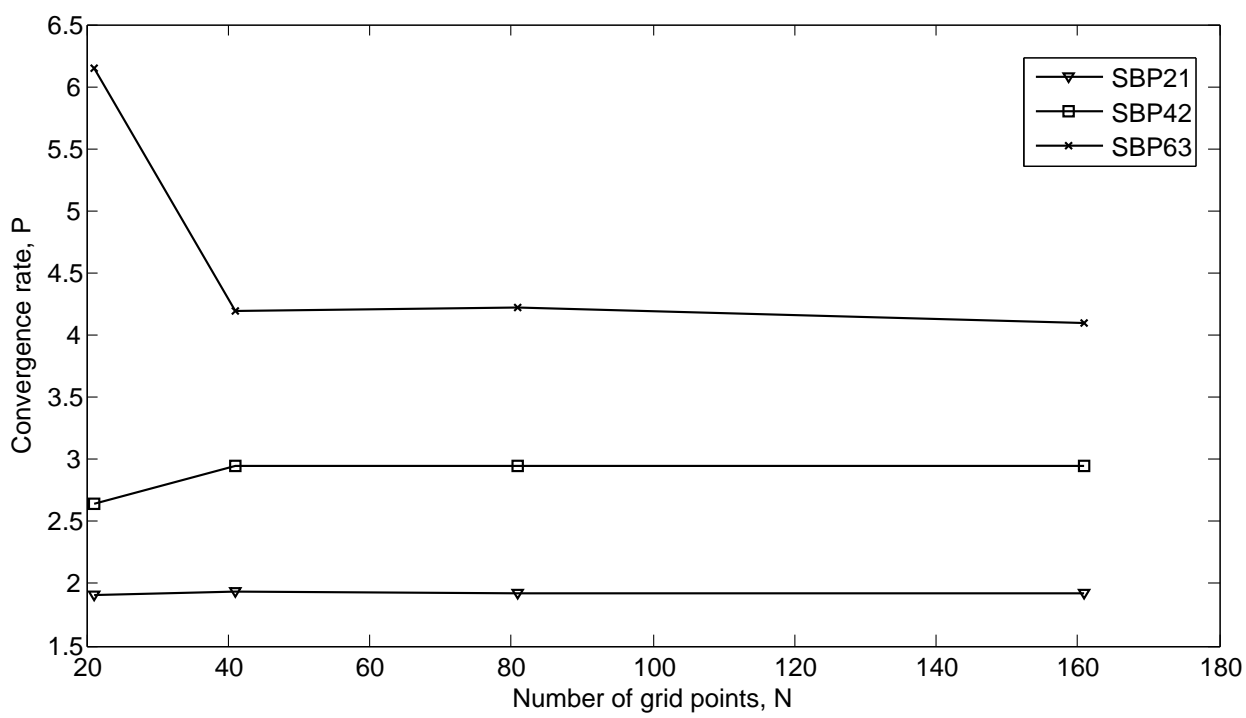

Figure 4. Mesh refinements and convergence rates, one-dimensional case, $t=1$.

Long time calculations for the one-dimensional case presented in Figure 5 show that the scheme is error bounded. ${ }^{16}$ The scheme is also tested for constant preservation up to $t=10000$. The constant is preserved which means that the GCL is fulfilled numerically.

\section{B. The two dimensional problem}

In the two dimensional example we consider the physical domain to be a portion of a ring-shaped geometry. One can transform the domain from Cartesian coordinates, $x-y$, into the Polar coordinates, $r-\phi$, where 


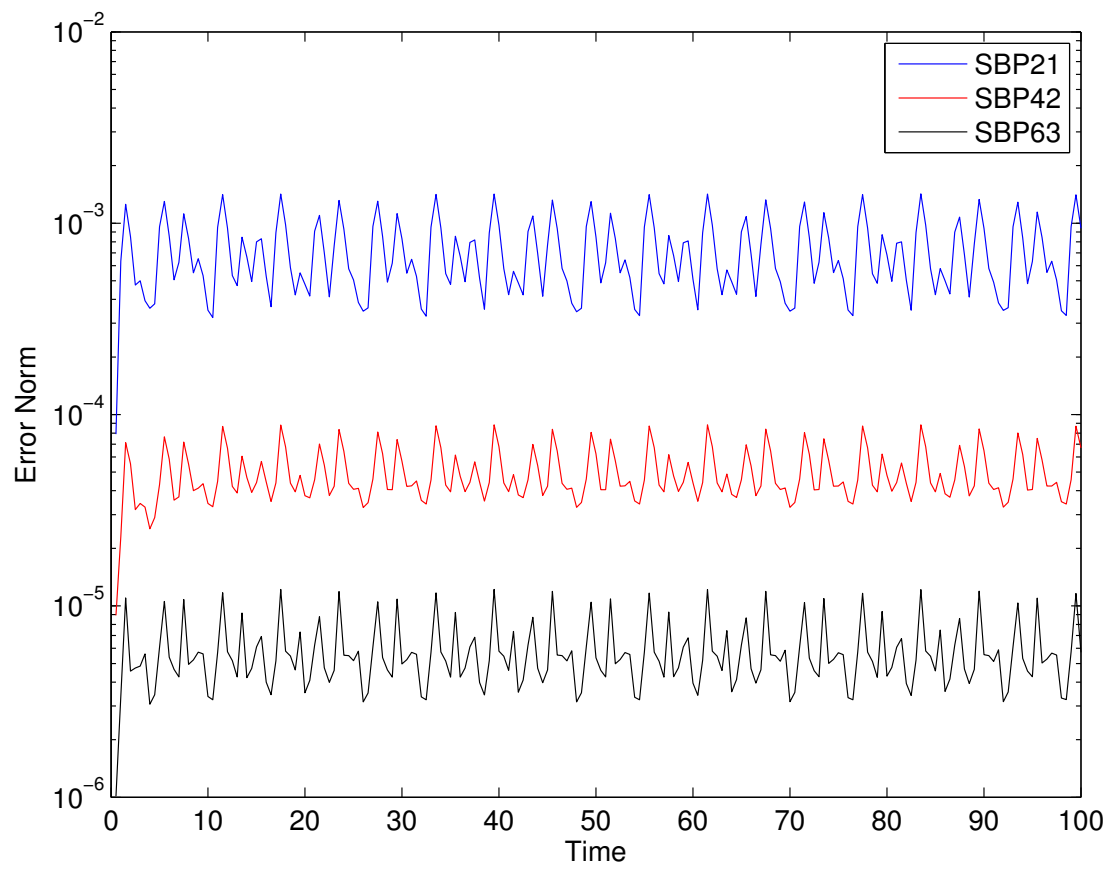

Figure 5. Long time calculations for one-dimensional case, error norm versus time.

$r=\sqrt{x^{2}+y^{2}}$ and $\phi=\tan ^{-1}\left(\frac{y}{x}\right)$. The domain is time dependent by $r_{0}(t) \leq r \leq r_{1}(t)$, and $\phi_{0}(t) \leq \phi \leq \phi_{1}(t)$, where the time dependency is chosen as

$$
\begin{array}{ll}
r_{0}(t)=1-\frac{0.1}{2 \pi} \sin (2 \pi t), & \phi_{0}=-\frac{0.5}{2 \pi} \sin (2 \pi t) \\
r_{1}(t)=2+\frac{0.2}{2 \pi} \sin (2 \pi t), & \phi_{1}=\frac{\pi}{2}-\frac{0.5}{4} \sin (2 \pi t),
\end{array}
$$

in which $r_{0}, r_{1}, \phi_{0}$ and $\phi_{1}$ are shown in Figure 6. The fixed coordinates are defined as

$$
\xi(x, y, t)=\frac{r(x, y, t)-r_{0}(t)}{r_{1}(t)-r_{0}(t)} \quad \eta(x, y, t)=\frac{\phi(x, y, t)-\phi_{0}(t)}{\phi_{1}(t)-\phi_{0}(t)} .
$$
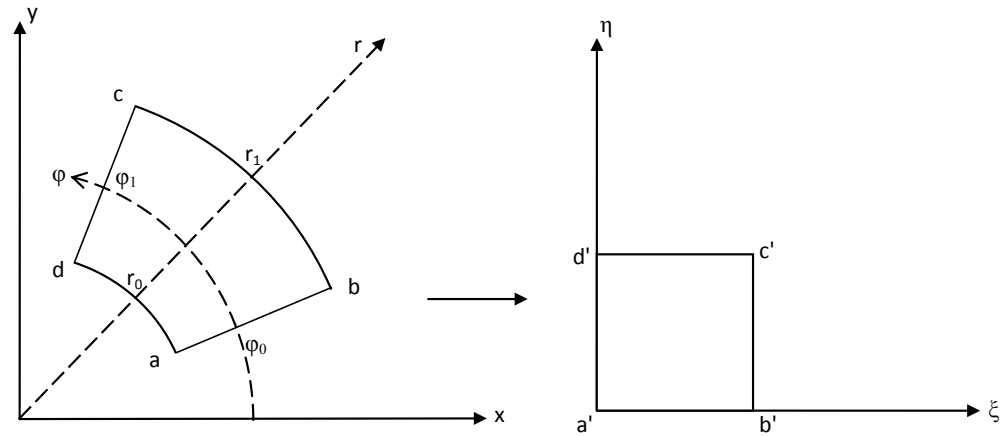

Figure 6. A schematic of the Cartesian-Polar transformation in the physical domain, the computational domain, and definition of $r_{0}, r_{1}, \phi_{0}$ and $\phi_{1}$.

A schematic of the physical and computational domain at different time steps is presented in Figure 7 and Figure 8 respectively. These schematics are for illustration purposes only, the numerical experiments are carried out on finer meshes with 51 grid points in each direction on the finest grid. Also we used $a=1$ and $b=1$. 


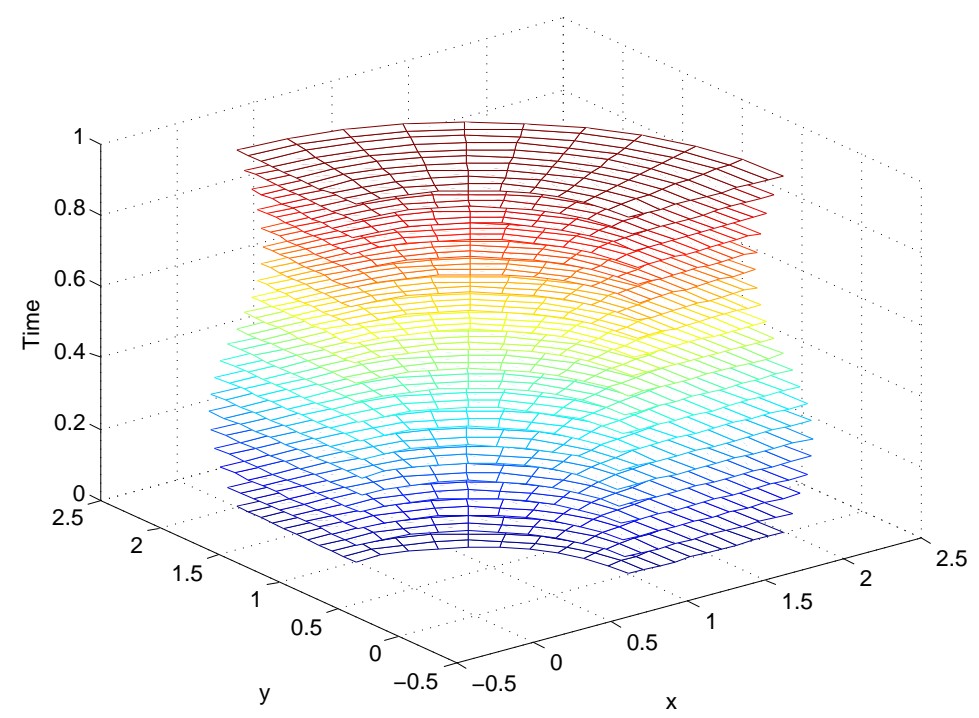

Figure 7. A schematic of the physical domain at different time steps.

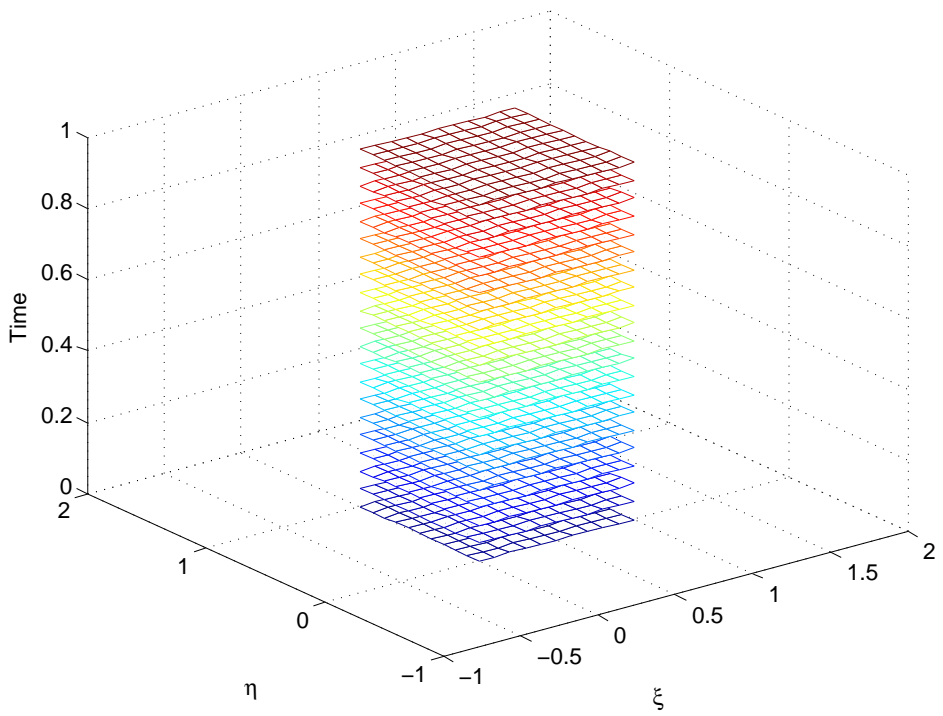

Figure 8. A schematic of the computational domain at different time steps.

In order to show that the scheme automatically selects the appropriate boundary procedure, the time dependency of the physical domain is changed to

$$
\begin{array}{ll}
r_{0}(t)=1-\frac{0.1}{2 \pi} \sin (2 \pi t), & \phi_{0}=-\frac{5}{2 \pi} \sin (2 \pi t) \\
r_{1}(t)=2+\frac{0.2}{2 \pi} \sin (2 \pi t), & \phi_{1}=\frac{\pi}{2}-\frac{5}{4} \sin (2 \pi t) .
\end{array}
$$

A schematic showing the physical domain at different time levels, and the penalty coefficient corresponding to the mid-point of the south boundary versus time are presented in Figure 9 and Figure 10 respectively. Note that (52) is only introduced to show the automatical implimentation of the south boundary procedure, and in other experiments (50) is used.

The convergence rate in the two dimensional case is calculated as 


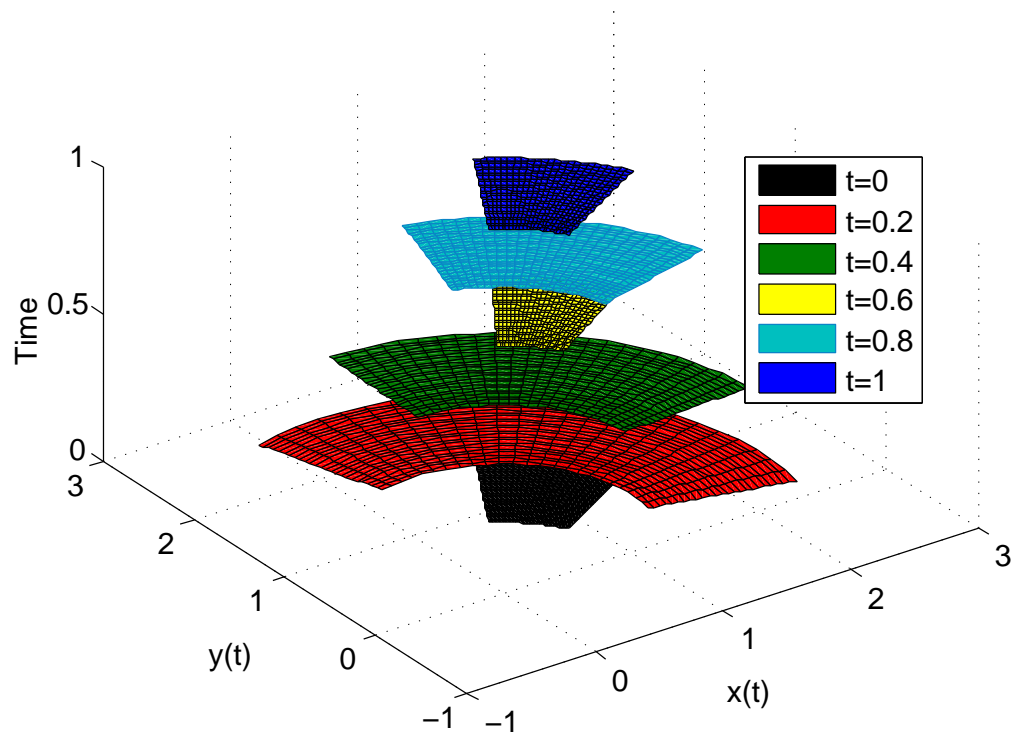

Figure 9. A schematic of the moving phycial domain at different time levels.

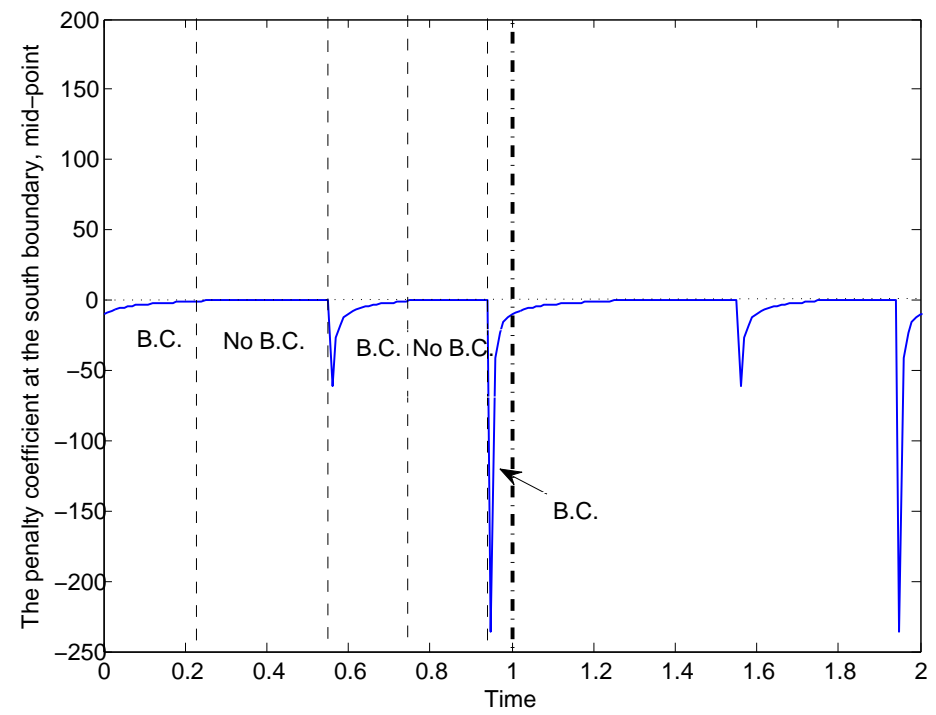

Figure 10. A schematic of the penalty coefficient at the mid-point of the south boundary versus time.

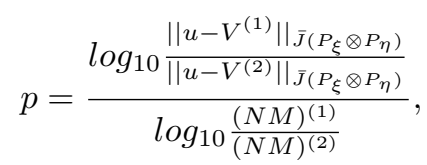

in which $u$ is the exact solution, $V^{(1)}$ and $V^{(2)}$ are the corresponding numerical solutions on the meshes of $(N M)^{(1)}$ and $(N M)^{(2)}$ grid points respectively.

The numerical solution in our experiments converges to the exact solution at $\mathrm{t}=1$ with the convergence rates presented in Figure 11. The convergence rates are in agreement with the theory mentioned above.

Long time calculations for the two-dimensional case is presented in Figure 12, and show that the scheme is error bounded. ${ }^{16}$ The error level corresponding to the sixth order operator will drop down if a fine enough mesh is used. Also in the two-dimensional case a constant is preserved up to $t=10000$, and the GCL is fulfilled. 


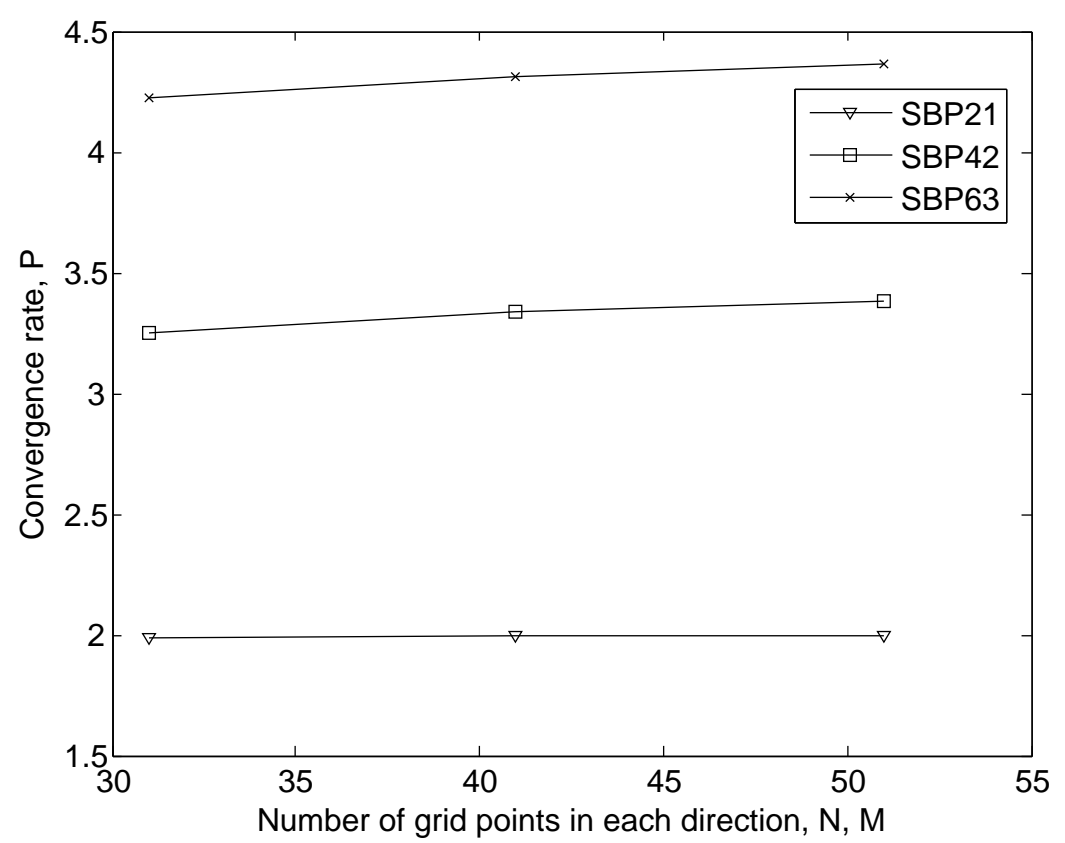

Figure 11. Mesh refinements and convergence rates, two-dimensional case, $\mathrm{t}=1$.

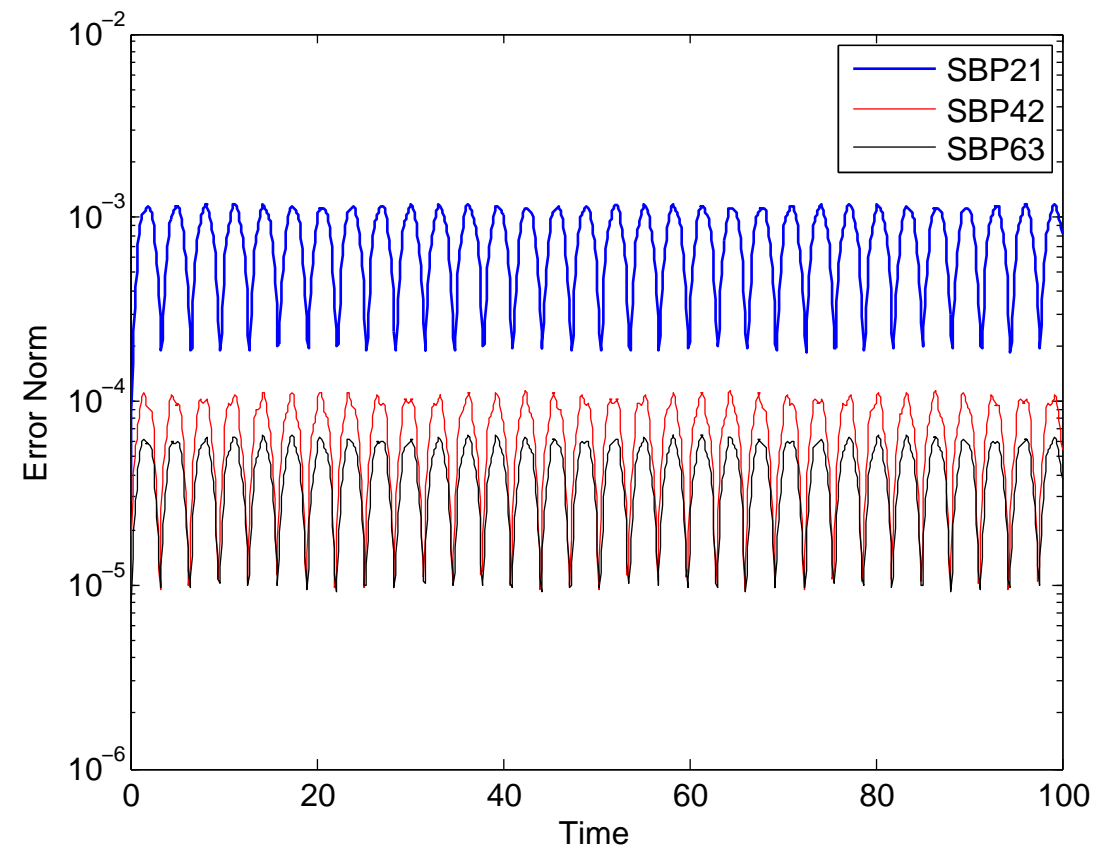

Figure 12. Long time calculations for two-dimensional case, error norm versus time.

\section{Conclusions and future work}

We consider constant coefficient one- and two-dimensional hyperbolic equations in time-dependent curvilinear coordinates. The equations are transformed into a fixed coordinate frame, resulting in variable coefficient hyperbolic equations. By applying the energy method to the transformed equations, we derive 
well-posed boundary conditions, leading to energy estimates for the continuous problem.

By using summation-by-parts operators, a special splitting technique, weak implementation of the boundary conditions, and the discrete energy method, stable and accurate numerical schemes are constructed. The discrete energy estimates for the one- and two-dimensional advection equations are similar to the continuous ones, but with added damping terms. Hence, the schemes are energy-stable. Furthermore, the Geometric Conservation Law is shown to hold, both theoretically and computationally.

Due to the moving boundaries, the number and location of the boundary conditions depend on the transformation relations. Penalty coefficients are derived such that the appropriate boundary procedure is automatically activated by the scheme.

The schemes are tested and the spatial accuracy agree with the theory for all cases considered. Long time calculations for one- and two-dimensional cases show that the energy rate of the solution is bounded by the data of the problem. Long time calculations also show that the schemes are error bounded.

Future work will include expansion of the current methodology to hyperbolic systems like the Euler equations and finally for incompletely parabolic systems like the Navier-Stokes equations.

\section{References}

\footnotetext{
${ }^{1}$ Fletcher, C. A. J., Computational Techniques For Fluid Dynamics, Vol. 2, Second Edition, 1991.

${ }^{2}$ Thomas, P.D., Lombard, C.K., Geometric Conservation Law and Its Application to Flow Computations on Moving Grids, AIAA Journal, Vol. 17, 1979.

${ }^{3}$ Abe, Y., Iizuka, N., Nonomura, T., Fujii, K., Symmetric-conservative Metric Evaluations for High-order Finite Difference Scheme with the GCL Identities on Three-dimensional Moving and Deforming Mesh, ICCFD7-2801, 2012.

${ }^{4}$ Nordström, J., Conservative Finite Difference Formulations, Variable Coefficients, Energy Estimates and Artificial Dissipation, Journal of Scientific Computing, Vol. 29, No. 3, December 2006.

${ }^{5}$ Strand B., Summation by Parts for Finite Difference Approximations of $d / d x$, Journal of Computational Physics, Vol. 110, No. 1, 1994.

${ }^{6}$ Carpenter M. H., Nordström, J., Gottleib D. A Stable and Conservative Interface Treatment of Arbitrary Spatial Accuracy, Journal of Computational Physics, Vol. 148, No. 2, 1999.

${ }^{7}$ Svärd, M. Nordström, J., On the Order of Accuracy for Difference Approximations of Initial Boundary Value Problems, Journal of Computational Physics, Vol. 218, No. 1, 2006.

${ }^{8}$ Kreiss, H. -O., Scherer, G., Finite element and finite difference methods for hyperbolic partial differential equations, Academic Press, New York, 1974.

${ }^{9}$ Nordström, J., Carpenter, H., Boundary and interface conditions for high order finite difference methods applied to the Euler and NavierStokes equations, Journal of Computational Physics 148, 621645, 1999.

${ }^{10}$ Nordström, J., Carpenter, H., High-order finite difference methods, multidimensional linear problems and curvilinear coordinates, Journal of Computational Physics 173, 149174, 2001.

${ }^{11}$ Nordström, J., Gong, J., Adamsson, C., Eliasson, P., A stable hybrid method for hyperbolic problems, Journal of Computational Physics 212, 436453, 2006.

${ }^{12}$ Nordström, J., Gustaffsson,R., High order finite difference approximations of electromagnetic wave propagation close to material discontinuities, Journal of Scientific Computing 18 (2), 215234, 2003.

${ }^{13}$ Nordström, J., Ham, F., Shoeybi, M., E. van der Weide, Svard, M., Mattsson, K., Iaccarino, G., Gong, J., A hybrid method for unsteady fluid flow, Computers and Fluids 38, 875882, 2009.

${ }^{14}$ Svärd, M., Carpenter, M. H., Nordström, J., A stable high-order finite difference scheme for the compressible NavierStokes equations: far-field boundary conditions, Journal of Computational Physics 225 (1), 10201038, 2007.

${ }^{15}$ Svärd, M., Nordström, J., A stable high-order finite difference scheme for the compressible NavierStokes equations: no-slip wall boundary conditions, Journal of Computational Physics 227 (10), 48054824, 2008.

${ }^{16}$ Nordström, J., Error bounded schemes for time-dependent hyperbolic problems, SIAM J. SCI. COMPUTE. Vol. 30, No.1m pp.46-59, 2007.
} 